\title{
Propiedades psicométricas del cuestionario de actitudes hacia la sexualidad en la vejez (Casv-10). Medellín-Colombia*
}

Psychometric properties of the questionnaire of attitudes towards sexuality in old age (Casv-10). Medellín Colombia Propriedades psicométricas do questionário de atitudes sobre a sexualidade na velhice (casv-10). Medellín-Colombia.

DOI: https://doi.org/10.21803/pensam.v11121-1.261

Edgardo Javier Ramos Caballero https://orcid.org/0000-0001-8545-5503

Ana del Carmen Acosta López https://orcid.org/0000-0002-8595-1520

Estela Melguizo Herrera https://orcid.org/0000-0002-8087-9718
¿Cómo citar este artículo?

Ramos, E., Acosta, A. \& Melguizo, E. (2018). Propiedades psicométricas del cuestionario de actitudes hacia la sexualidad en la vejez (Casv-10). Medellín-Colombia. Pensamiento Americano,
$11(22), 40-49$.

DOI: https://doi.org/10.21803/pensam.v11i21-1.261

\begin{abstract}
Resumen
Introducción: El estudio de la sexualidad requiere de instrumentos válidos y confiables. Objetivo: Valorar las propiedades psicométricas del Cuestionario de Actitudes hacia la Sexualidad en la Vejez CASV10, Medellín-Colombia. Método: Estudio de validación. Participaron 300 adultos. Se calculó consistencia interna; dimensionalidad; validez de constructo y análisis de funcionamiento diferencial de los ítems. Resultados: Alfa de Cronbach de 0,796. La dimensionalidad identificó tres dominios que dieron cuenta del $40,61 \%$ de la varianza total. La validez del constructo del modelo que se probó en el análisis factorial confirmatorio correspondió a la solución factorial del análisis factorial exploratorio y mostró una correlación del error de la covarianza: $x 2$ : 59,149; gl: 29; raíz cuadrada del error medio de aproximación: 0,059, IC 90\%: 0,037 - 0,080); índice comparativo de ajuste: 0,982 e índice de Tucker-Lewis: 0,972. Se probó la invarianza factorial de acuerdo con sexo obteniendo los siguientes índices de ajuste: x2: 147,659; gl: 29; raíz cuadrada del error medio de aproximación: 0,117, IC 90\%: 0,098 - 0,136); índice comparativo de ajuste: 0,998 e índice de Tucker-Lewis: 0,997. El análisis de funcionamiento diferencial de los ítems para todos los reactivos mostró un $\mathrm{p}$-valor $>0,05$. Conclusión: Es una escala de tres dimensiones confiables.
\end{abstract}

PALABRAS CLAVES: Sexualidad, validez, estudios de validación, adultos (DeCS).

\begin{abstract}
Introduction: The study of sexuality requires valid and reliable instruments. Objective: To assess the psychometric properties of the Attitudes Questionnaire towards Sexuality in Old Age CASV-10, MedellínColombia. Method: Validation study. 300 adults participated. Internal consistency; dimensionality; construct validity and differential functioning analysis of the items were calculated. Results: Cronbach's alpha of 0.796. The dimensionality identified three domains that accounted for $40.61 \%$ of the total variance. The validity of the model construct that was tested in the confirmatory factorial analysis corresponded to the factorial solution of the exploratory factorial analysis and showed a correlation of the error of the covariance: 2: 59,149; gl: 29; square root of the average approximation error: $0.059,90 \% \mathrm{Cl}$ : $0.037-0.080$ ); comparative index of adjustment: 0.982 and Tucker-Lewis index: 0.972. The factorial invariance was tested according to sex, obtaining the following adjustment indexes: x2: 147.659; gl: 29; square root of the average approximation error: 0.117, 90\% Cl: 0.098-0.136); comparative index of adjustment: 0.998 and TuckerLewis index: 0.997. The analysis of differential functioning of the items for all the reagents showed a $p$-value $>0.05$. Conclusion: It is a scale of three reliables dimensions.
\end{abstract}

KEYWORDS: Sexuality, validity, validation studies, adults (DeCS).

\footnotetext{
* Artículo de reflexión producto del proyecto de investigación "Las Políticas Públicas como Garantía de Derechos Humanos en Colombia", inscrito en la Dirección de Investigaciones de la Universidad Pedagógica y Tecnológica de Colombia -UPTC y vinculado al grupo de investigación Primo Levi de la Facultad de Derecho y Ciencias Sociales de la UPTC.
} 


\section{Resumo}

Introdução: O estudo da sexualidade requer de instrumentos válidos e confiáveis. Objetivo: valorar as propriedades psicométricas do Questionário de Atitudes sobre a Sexualidade na velhice CASV-10, em Medellín, na Colômbia. Método: Estudo de validação. Participaram 300 adultos. Calculou-se a consistência interna, a dimensionalidade, a validez do constructo e a análise do funcionamento diferencial dos itens. Resultados: Alfa de Cronbach de 0,796. A dimensionalidade identificou três domínios que deram conta de 40,61\% da variação total. A validez do constructo do modelo que se provou na análise fatorial confirmatória correspondeu à solução fatorial de análise fatorial exploratória e mostrou uma correlação de erro de covariância X2: 59,149; gl: 29; raiz quadrada de erro médio de aproximação: 0,059, IC 90\%: 0,037 - 0,080; índice comparativo de ajuste: 0,982 e índice de Tucker-Lewis: 0,972. Se provou a invariância fatorial de acordo com o sexo obtendo os seguintes índices de ajuste: x2: 147,659; gl: 29; raiz quadrada de erro médio de aproximação: 0,117, IC 90\%: 0,098 - 0,136; índice comparativo de ajuste: 0,998 e índice de Tucker-Lewis: 0,997. A análise de funcionamento diferencial dos itens para todos os reativos mostrou um p-valor>0,05. Conclusão: É uma escala de três dimensões confiáveis.

Palavras-chave: Sexualidade; Velhice; Estudos de Validade; Adultos (DeCS).

\section{Perfil}

Enfermero. Doctorando en Ciencias Biomédicas del IUNIR. Docente Asociado de la Corporación Universitaria Adventista. Medellín, Colombia.

ejramos@unac.edu.co.

\section{Perfil}

Enfermero, Docente y subgerente científica de enfermería de la Universidad de Cartagena, Cartagena-Colombia, Correo electrónico: aacostal@ unicartagena.edu.co.

\section{Perfil}

Docente titular Facultad de Enfermería de la Universidad de Cartagena, Cartagena-Colombia, emelguizoh@unicartagena.edu.co.

Edgardo Javier Ramos Caballero

Magister en Salud Pública.

\section{Ana del Carmen Acosta López}

Especialista en enfermería medicoquirurgica con énfasis en cuidados intensivo

\section{Estela}

Melguizo Herrera

Ph.D en enfermería 


\section{Introducción}

L

a Organización Mundial de la Salud (OMS) define la sexualidad humana, como la "integración de los aspectos somático, intelectual, emocional y social por los que se enriquecen y potencian la personalidad y el amor" (Rodríguez, 2008, p.3); hace parte importante en el desarrollo evolutivo, donde el potencial y el disfrute sexual se mantienen a lo largo de la vida, (Orozco y Rodríguez, 2006) y además influye positivamente en la salud y en las relaciones interpersonales (Nieva et al., 2010).

Abella y Castro (2014), afirma en su estudio que se sigue manteniendo el deseo y la actividad sexual en la tercera edad, más en varones que en mujeres, aunque disminuye la frecuencia y se manifiesta de diferentes formas que en otras edades.

Sin embargo, a pesar de ser un tema estudiado y explorado por la sociedad, los mismos adultos mayores y los profesionales de la salud desconocen muchos aspectos de esto, presentándose dudas y creándose mitos al respecto (González, J. M. \& González, J., 2005).

Para Esguerra (2007), el que los adultos mayoresse mantengan sexualmente activos influye en su calidad de vida y es un indicador de envejecimiento exitoso. Por otro lado, para Melguizo et al., (2015) es importante contar con instrumentos validados para el que hacer de los profesionales de la salud facilitando el trabajo y la intervención que realizan con los adultos mayoresy por medio de estos tener un acercamiento confiable a la realidad de los fenómenos atendidos.

Dentro de la literatura revisada en México, Orozco y Rodríguez (2006), Propusieron un cuestionario acerca de las actitudes hacia la sexualidad en la vejez (CASV), producto de la revisión de literatura e investigaciones al respecto que reportaban un vacío de información sobre dichas actitudes y perjuicios.

En Floridablanca, Colombia, Cerquera, Galvis y Cala (2012) utilizó el mismo CASV adaptado al contexto, quedando conformado por 16 ítems con escala de respuesta dicotómica y reportó que el cuestionario era confiable. En Cartagena, Colombia, Melguizo et al. (2015) validó el CASV, en su versión original, en adultos mayores de 60 años, mostrando una consistencia interna con altos coeficientes en la primera y la segunda aplicación, alta estabilidad y un intervalo de confianza del 95\%.

El objetivo de este estudio fue valorar las propiedades psicométricas del CASV-10 en la ciudad de Medellín, Colombia.

\section{MÉTODO:}

Diseño y población de estudio: se diseñó un estudio de validación o de evaluación de pruebas sin criterio de referencia (CampoArias \& Oviedo, 2008) (Campo-Arias, Herazo \& Oviedo, 2012; Presser et al., 2004; y Pineda, Alvarado \& Canales, 1994). La población estuvo conformada por personas mayores de 18 años. La muestra fueron 300 personas, sin condición de discapacidad mental o física. Se llevó a cabo un muestreo no probabilístico, por conveniencia. El número de participantes fue suficiente para los distintos tipos de prueba que se llevaron a cabo. La edad de los participantes estuvo entre 18 y 88 años y un promedio de edad de 46 años. La tabla No. 1 ilustra sobre las características de los participantes. 
Tabla 1.

Características Sociodemográficas de los Participantes

\begin{tabular}{|c|c|}
\hline Variable & $\%(n)$ \\
\hline \multicolumn{2}{|l|}{ Sexo } \\
\hline Masculino & $21,3 \%(64)$ \\
\hline Femenino & $78,6 \%(236)$ \\
\hline \multicolumn{2}{|l|}{ Escolaridad } \\
\hline Ninguna & $1 \%(3)$ \\
\hline Primaria & $13 \%(39)$ \\
\hline Bachiller & $19 \%(57)$ \\
\hline Técnica & $9 \%(27)$ \\
\hline Univeritario & $46 \%(138)$ \\
\hline Posgrados & $12 \%(36)$ \\
\hline \multicolumn{2}{|c|}{ Estrato Socioeconómico } \\
\hline I & $5 \%(15)$ \\
\hline II & $13 \%(39)$ \\
\hline III & $30 \%(90)$ \\
\hline IV & $23 \%(69)$ \\
\hline $\mathrm{V}$ & $28 \%(84)$ \\
\hline VI & $1 \%(3)$ \\
\hline \multicolumn{2}{|l|}{ Estado Marital } \\
\hline Con pareja estable & $39 \%(117)$ \\
\hline Sin pareja estable & $61 \%(183)$ \\
\hline
\end{tabular}

Fuente: Encuestas aplicadas.

Instrumento: el cuestionario CASV, fue propuesto por Orozco y Rodríguez (2006); consta, en su versión original de 14 preguntas y las posibilidades de respuestas van desde completamente verdadero (4) hasta completamente falso $(0)$, con una calificación tipo Likert.

El cuestionario fue validado en Cartagena, Colombia,conungrupode130adultosmayores y reportó una consistencia interna alta en la primera medición, con un alfa de Chronbach igual a 0,83 y un Omega de McDonald de 0,87; y en la segunda medición reportó un alfa de Chronbach de 0,85 y un Omega de McDonald de 0,89. El análisis factorial exploratorio mostró dos factores relevantes (prejuicios y limitaciones) que explicaron el $42,6 \%$ de la varianza. El Funcionamiento Diferencial de los Ítems, mostró coeficientes adecuados, con excepción del ítem 14, que presentó valor alto $(t=0,37)$. El CASV presento alta estabilidad $(r=0,82$ y $\mathrm{CCl}=0,89$; intervalo de confianza del 95\% [IC95\%], 0,83-0,92; $\mathrm{p}<$ 0,001) (Melguizo et al., 2015).

Cerquera et al. (2012), en Floridablanca, Colombia, el CASV fue adaptado por otros investigadores y "quedó conformado por 16 ítems, con escala de respuesta dicotómica, cuyas opciones son sí o no". En este caso, se aplicó el cuestionario a 328 personas: 107 adolescentes, 68 adultos y 153 personas mayores. La validación del cuestionario reveló un coeficiente KR-20 de 0,592, con un error estándar de medición equivalente al 1,43 y una desviación estándar de puntajes de 2,24; mostrando que el cuestionario se aceptaba como confiable.

Para este estudio en la ciudad de Medellín, el CASV fue revisado por un equipo de tres profesionales con experiencia en investigación, validación de instrumentos y en el cuidado de personas mayores, quienes contribuyeron a contextualizarlo de acuerdo con el imaginario colombiano (Corral, 2009; Labrador, 2006); considerando pertinente que se plantearan todas las preguntas en forma positiva y que se eliminaran cuatro preguntas que parecían repetir información. Para este caso el CASV quedó conformado por 10 ítems (CASV-10): 8 ítems indagan los prejuicios sociales sobre la vejez y la sexualidad y 2 cuestionan las enfermedades resultantes del proceso del envejecimiento. Se excluyeron 
los ítems 1, 5, 13 y 14 del cuestionario original. Adicionalmente, los ítems 4, 6, 7, 8 y 12 fueron redactados con un vocabulario acorde al contexto de la ciudad de Medellín.

Para los puntajes de esta escala se siguió lo propuesto para los estudios de actitudes, según lo estipulado por los autores de la escala (8); donde las posibilidades de respuesta son:

- $\quad$ Completamente verdadero: 4 puntos.

- Verdadero: 3 puntos.

- Ni falso, ni verdadero: 0 puntos

- Falso: 2 puntos.

- Completamente falso: 1 punto.

Las puntuaciones totales están entre 0 y 40; y a mayor puntuación se consideran actitudes más prejuiciosas o negativas; es decir aquellas que tienen una calificación de "completamente verdadero". La tabla 2 , ilustra los ítems considerados para esta investigación y como se resumieron.
Análisis Estadístico: inicialmente se determinó la factorizabilidad de la matriz con la prueba de esfericidad de Barlett y la prueba de adecuación de la muestra de Kaiser-Meyer-Olkin (KMO). La prueba de Bartlett es un estadístico de alta sensibilidad y es apropiada si muestra alto x2 y p-valor <0,05. El KMO es una prueba más conservadora, que se entiende aceptable si muestra un valor >0,70. (Costello \& Osborne, 2005).

Posteriormente, se realizó análisis factorial exploratorio (AFE) para conocer la estructura de factores empleando el método de factores principales. El número de factores a extraer se determinó a través de los valores propios (>1.0), sin embargo, este criterio ha mostrado ser el menos preciso; así, se empleó un gráfico de sedimentación de Catell. Para definir la conformación de los factores, se tomaron en cuenta las cargas factoriales $\geq 0,40$. Se empleó rotación oblicua (promax), puesto que se consideró que los posibles

Tabla 2.

Items para evaluar las actitudes sobre sexualidad en la vejez.

\begin{tabular}{ll}
\hline Item & Abreviado \\
\hline La menopausia marca el final de la vida sexual de la mujer & Menopausia \\
Los ancianos que demuestran el amor se ven ridículos & Ridículo \\
Los ancianos no tienen vida sexual activa & Inactivo \\
Los ancianos son impotentes & Impotencia \\
Las ancianas son frías para el sexo & Frialdad \\
Los ancianos ya no tienen deseos sexuales & No deseo \\
Los cambios que trae el envejecimiento impiden tener actividad & Vejez \\
sexual & \\
Las enfermedades más frecuentes en la vejez limitan la actividad & Enfermedad \\
sexual & Tratamiento \\
Los tratamientos médicos en la vejez complican la actividad sexual & Juventud \\
La belleza y la sexualidad son para los jóvenes &
\end{tabular}

Fuente: Construcción de los investigadores. 
factores estarían correlacionados entre sí (De la Fuente, 2011).

Para proceder con el análisis factorial confirmatorio (AFC) y determinar validez de constructo, inicialmente se verificó la normalidad multivariable con el test de Mardia. Aunque no se encontró normalidad multivariable, el AFC es robusto para este tipo de supuestos cuando se tienen tamaños de muestra amplios (>200) y no hay normalidad multivariable. El AFC se realizó para confirmar la estructura de factores previamente determinado en el AFE con el método de estimación Mínimos Cuadrados Parciales con Media y Varianza Ajustada (MCPMVA). Para la evaluación del ajuste del modelo, se determinaron los siguientes estadísticos: $x 2$, grados de libertad ( $\mathrm{gl}$ ), raíz cuadrada del error medio de aproximación (RCEMA) e intervalo de confianza al 90\% (IC 90\%), índice comparativo de ajuste (ICA) e índice de Tucker-Lewis (ITL). La evaluación de estos estadísticos (ajuste del modelo), se realizó empleando los criterios propuestos por $\mathrm{Hu}$ \& Bentler (Simanca, Fortich \& González, 2017) así: p-valor para $\times 2>0,05, R C E M A \leq 0,06$, ICA e $I T L \geq 0,95$. Asimismo, empleando AFC con los mismos criterios de ajuste del modelo, se determinó la invarianza factorial del constructo de acuerdo a sexo.

La consistencia interna se determinó a través del coeficiente alfa de Cronbach, considerándolo como aceptable cuando el estadístico fuera >0,70 (Cronbach, 1951).

El análisis de Funcionamiento Diferencial de los Ítems (FDI) se realizó mediante el coeficiente de correlación tau de Kendall. Este coeficiente arroja valores entre $-1 \mathrm{y}+1$. Se consideró la presencia de desempeño diferencial cuando se encontraron valores $>0,30$, sin importar el signo del coeficiente y el valor de probabilidad, dado que se contó con una muestra >100 participantes (Kendall, 1938).

Aspectos éticos: Se obtuvo la aprobación de un comité institucional de ética en investigación. Se dio a conocer a los participantes el objetivo del estudio y firmaron todos los consentimientos informados. Según la Resolución 008430 del Ministerio de Salud de Colombia (1993), esta investigación representó un riesgo mínimo para los participantes ya que no se llevaron a cabo acciones dañinas para la integridad física o emocional de los participantes.

\section{RESULTADOS:}

Consistencia Interna: EI CASV-10, mostró globalmente coeficiente alfa de Cronbach de 0,796. Las subescalas, mostraron 0,776; 0,649 y 0,6452 respectivamente.

Dimensionalidad: El inició del análisis de factores mostró indicadores aceptables ( $\mathrm{KMO}=0,83$; prueba de esfericidad de Barlett $\times 2=732,34$, grados de libertad $=45$; $\mathrm{p}$-valor $<0,001)$. En la mejor solución de factores se identificaron tres dominios relevantes que dieron cuenta del 40,61\% de la varianza total; el primero Prejuicios se conformó con los ítems 1, 2 y 10, el segundo denominado Mitos con los ítems 3 al 6 y el tercero denominado Limitaciones con los ítems 7 al 9. Las comunalidades se hallaron entre 0,2981 y 0,4987 y las cargas factoriales entre 0,4018 y 0,7146. La comunalidad y la carga factorial para cada reactivo se muestran en la Tabla 3. 
Tabla 3.

Comunalidades y cargas factoriales para cada reactivo del CASV-10.

\begin{tabular}{lcccc}
\hline Ítem & Factor & Factor & Factor & Comunalidad \\
& I & II & III & \\
\hline Menopausia & 0,6039 & & & 0,3787 \\
Ridículo & 0,6516 & & & 0,3842 \\
Inactivo & & 0,4018 & & 0,4384 \\
Impotencia & & 0,6240 & & 0,4987 \\
Frialdad & & 0,6988 & & 0,4800 \\
No deseo & & 0,7146 & & 0,4881 \\
Vejez & & & 0,4027 & 0,3234 \\
Enfermedad & & & 0,6196 & 0,4506 \\
Tratamiento & & & 0,6023 & 0,3247 \\
Juventud & 0,4900 & & & 0,2981 \\
Valor Propio & 1,9641 & 2,4908 & 1,8403 & \\
Varianza Explicada (\%) & 6,42 & 29,26 & 4,94 & \\
Alfa de Cronbach & 0,776 & 0,649 & 0,6452 & \\
\hline
\end{tabular}

Fuente: Construcción de los investigadores

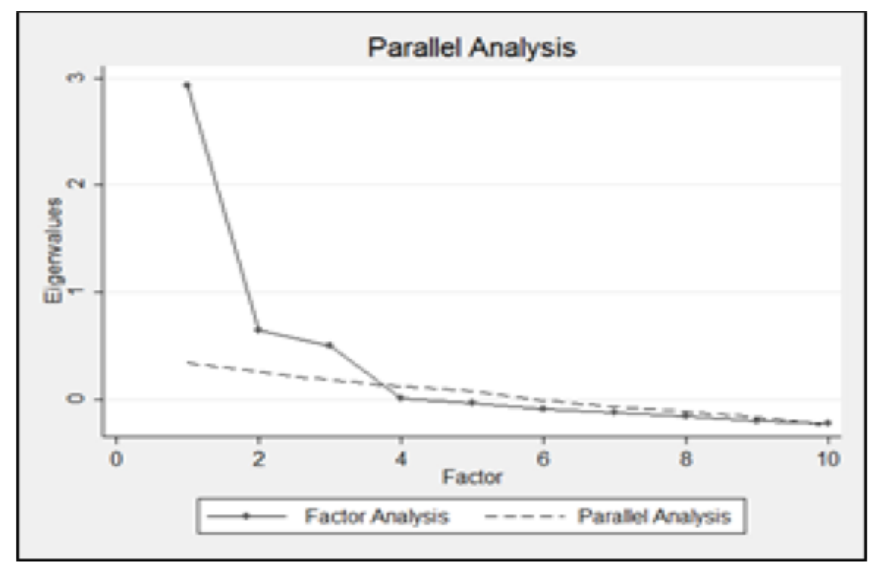

Gráfico 1: Gráfico de Catell que indica la sugerencia de retener 3 factores dados la intersección entre las dos líneas del gráfico.

Fuente: Construcción de los investigadores 
Validez de Constructo: El modelo que se probó en AFC correspondió a la mejor solución factorial en el AFE y arrojó los siguientes estadísticos de ajuste después de aplicar tres índices de modificación (correlación del error de la covarianza): x2: 59,149; gl: 29; RCEMA: 0,059, IC 90\%: 0,037 - 0,080); ICA: 0,982 e ITL: 0,972 .

Invarianza Factorial: Teniendo en cuenta el modelo ajustado derivado del análisis de validez de constructo, se probó invarianza factorial de acuerdo a sexo obteniendo los siguientes índices de ajuste: x2: 147,659; gl: 29; RCEMA: 0,117, IC 90\%: 0,098 - 0,136); ICA: 0,998 e ITL: 0,997.

Funcionamiento Diferencial de los Ítems: La tabla 4, se muestra el análisis de funcionamiento diferencial de los ítems para los reactivos de la escala; Todos tuvieron p-valor $>0,05$.

Tabla 4.

Análisis de Funcionamiento Diferencial de los İtems.

\begin{tabular}{ll}
\hline Item & $\boldsymbol{\tau}$ de Kendall \\
\hline Menopausia & $-0,0347$ \\
Ridículo & $-0,0551$ \\
Inactivo & 0,0404 \\
Impotencia & 0,0929 \\
Frialdad & 0,0437 \\
No deseo & 0,0349 \\
Vejez & $-0,0089$ \\
Enfermedad & $-0,0624$ \\
Tratamiento & $-0,0221$ \\
Juventud & $-0,0957$ \\
\hline
\end{tabular}

Fuente: Encuestas aplicadas.

\section{DISCUSIÓN}

Los resultados evidenciaron que CASV-10 es una escala válida y confiable en una muestra de adultos de la ciudad de Medellín, Colombia.
Aunque en el estudio realizado por Cerquera et ál. (2012), se calculó la consistencia interna a través de la fórmula 20 de KuderRichardson, por ser una escala opción de respuesta dicotómica; en el presente estudio se calculó a través del Alfa de Cronbach, por ser una versión de la escala con respuestas politómica, manteniendo la propuesta de la versión original; estos coeficientes tienen equivalencia matemática (Campo-Arias \& Oviedo, 2008).

Se evidenció una buena consistencia interna en todos los contextos estudiados, pero se obtuvieron más altos coeficientes en Cartagena con 0,83 (Melguizo et al., 2015) y Medellín 0,796. Quizá estos resultados se relacionen con el hecho de que las versiones de la escala aplicadas en Cartagena y Medellín hayan sido más similares entre sí en cuanto al número de opciones de respuesta; mientras que en la versión aplicada en Floridablanca la opción de respuesta fue dicotómica y se agregaron dos ítems a la escala original. Este hallazgo es congruente con lo que afirma la literatura a este respecto (Campo-Arias \& Oviedo, 2008).

Por otra parte, la literatura afirma que el tamaño de la muestra de losestudios noafecta el resultado del cálculo de la consistencia interna de una escala, lo cual es similar a lo observado al comparar los resultados entre los estudios de Cartagena (130 participantes), Floridablanca (328 participantes) y Medellín (300 participantes) (Campo-Arias \& Oviedo, 2008; Paniagua, 2015).

En la investigación, la solución de factores se identificaron tres dominios relevantes que dieron cuenta del 40,61\% de la varianza total: prejuicios, mitos y limitaciones. Esto podría explicarse al contemplar que se trata de un instrumento poco usado y que para esta investigación fue modificado al eliminarse 
algunos ítems y redactarse otros en forma diferente; según Campo et al. (2012) afirma que pueden ser aceptables o manejables con precaución varianzas totales entre el 35 $y$ el $40 \%$.

Los hallazgos de esta investigación son similares a la propuesta original de Orozco y Rodríguez solo que en esta ocasión se omitió la pregunta con respecto al derecho de los adultos mayores al amor y a la vida sexual. Aunque Orozco y Rodríguez (2006) no se presentan datos sobre un análisis de factores que sustente ese supuesto.

Con respecto a las dimensiones del instrumento evidenciadas por el análisis de factores, estos hallazgos difieren de lo reportado por Melguizo et al. (2015), quien a pesar de conservar la versión original del cuestionario CASV, evidenció solo dos dimensiones relevantes: prejuicios y limitaciones.

Al considerar las comunalidades y los coeficientes del análisis de factores, parece que no hay necesidad de revisar la pertinencia de los ítems dentro del cuestionario (Campo-Arias \& Oviedo, 2008).

\section{CONCLUSIONES:}

Se concluye que el CASV-10 es una escala válida y confiable en adultos mayores en una muestra de adultos de la ciudad de Medellín, Colombia. Es una escala más breve y por lo tanto se espera que sea más fácil de aplicar en esta población de adultos mayores. Sin embargo, se requieren más investigaciones en otros contextos o con una muestra representativa de los adultos mayores de Medellín para corroborar el comportamiento psicométrico de este instrumento que pretende facilitar al personal de salud el abordaje de un aspecto importante en la vida de las personas como lo es la sexualidad.

\section{Referencias}

Abella, J. \& Castro, P. (2014). Percepción de la sexualidad en la vejez y las repercusiones en su práctica a través del estudio de las opiniones de tres grupos etarios: adolescentes, adultos y personas Mayores. (Trabajo de grado, pregrado). Universidad de Zaragoza, España. Recuperado de: http://zaguan.unizar. es/record/14977? Ln=es

Campo-Arias, A., \& Oviedo, H. (2008). Propiedades Psicométricas de una Escala: la Consistencia Interna. Revista de Salud Pública, 10 (5), 831-839.

Campo-Arias, A., Herazo. E. \& Oviedo, H. (2012). Análisis de factores: fundamentos para la evaluación de instrumentos de medición en salud mental. Revista Colombiana de Psiquiatría 41(3), 659-71.

Cerquera, A., Galvis, M. \& Cala, M. (2012). Amor, sexualidad e inicio de nuevas relaciones en la vejez: percepción de tres grupos etarios. Psychologia, 6(2), 73-81. Recuperado de: http://www.scielo.org.co/pdf/ psych/v6n2/v6n2a07.pdf

Corral, Y. (2009). Validez y confiabilidad de los instrumentos de la investigación para la recolección de datos. Revista ciencias de la Salud. 19(33). Recuperado de: http://servicio.bc.uc.edu.ve/educacion/ revista/n33/art12.pdf

Costello, A. B. \& Osborne, J. W. (2005). Best practices in exploratory factor analysis: Four recommendations for getting the most from your analysis. Pract Assess Researsh \& Evaluation. 10(7). Recuperado de: https://pareonline.net/pdf/v10n7.pdf.

Cronbach, J. (1951). Coefficient alpha and the internal structure of test. Psychometrika 16(2) 97-334.

De la Fuente, S. (2011). Análisis Factorial. Universidad Autónoma de Madrid.

Esguerra, I. (2007). Sexualidad después de los 60 años. Av. Enferm XXV (2), 124- 140. Recuperado de: http:// 
www.scielo.org.co/pdf/aven/v25n2/v25n2a12.pdf

González, J. M. \& González, J. (2005). Estudio descriptivo de la sexualidad en personas mayores de 60 años en el Caribe colombiano. Revista Psicogente R (13), 17-27. Recuperado de https://dialnet.unirioja.es/descarga/articulo/6113918.pdf

Kendall, M. (1938). A new measure of rank correlation. Biometrika. 30, 81-9.

Labrador, M. E. (2006). Formato para Validación de Instrumentos. Bárbula, Venezuela: Facultad de Odontología de la Universidad de Carabobo.

Melguizo, E., Álvarez, Y., Cabarcas, M., Calvo, R., Floréz, J., Moadie, O. \& Campo, A. (2015). Validez y confiabilidad del cuestionario de actitudes hacia la sexualidad en la vejez en adultos mayores en Cartagena, Colombia. Revista Colombiana de Psiquiatría 44(2), 87-92.

Ministerio de Salud de Colombia. (1993). Resolución 008430, Por la cual se establecen las normas científicas, técnicas y administrativas para la investigación en salud. Recuperado de: https://www.minsalud.gov.co/sites/rid/Lists/BibliotecaDigital/RIDE/DE/ DIJ/RESOLUCION-8430-DE-1993.PDF

Nieva, R., Velasco, J., Andrade, H., Rodríguez, C., Gonzales, S. \& Mendoza, M. (2010). Expresión de la conducta sexual en adultos mayores usuarios de una unidad de medicina familiar. Atención Familiar, 17 (1), 22-23. Recuperado de: http://www.medigraphic. com/pdfs/atefam/af-2010/af101f.pdf

Orozco, I. \& Rodríguez, D. (2006). Prejuicios y actitudes hacia la sexualidad en la vejez. Psicología y Ciencia Social, 8 (1), 3-10. Recuperado de: http://www. redalyc.org/pdf/314/31480101.pdf

Paniagua, R. (2015). Metodología para la validación de una escala o instrumento de medida. Universidad de Antioquia, facultad nacional de salud pública. Recuperado de: http://www.udea.edu.co/wps/ wcm/connect/udea/d76a0609-c62d-4dfb-83dc5313c2aed2f6/METODOLOG\%C3\%8DA+PARA+LA+VALIDACI\%C3\%93N+DE+UNA+ESCALA.pdf?MO$D=A J P E R E S$

Pineda, E., Alvarado, E. \& Canales, F. (1994). Metodología de la Investigación ( $2^{\mathrm{a}}$ ed.). Washington D.C: Organización Panamericana de la Salud. Recuperado de: http://187.191.86.244/rceis/registro/Metodologia\%20de\%20la\%20Investigacion\%20Manual\%20 para\%20el\%20Desarrollo\%20de\%20Personal\%20 de\%20Salud.pdf

Presser, S., Couper, M., Lessler, J., Martin, E., Martin, J., Rothgeb, J. (2004). Methods for testing and evaluating survey questions. Public Opin Q.68, 109-30. Recuperado de https://www.psc.isr.umich.edu/pubs/ abs/2405

Rodríguez, C. (2008). La sexualidad en el anciano. INEFOC. Recuperado de: http://www.acpgerontologia.com/documentacion/sexualidadrodriguezbenito.pdf

Simanca, M., Fortich, N. \& González, F. (2017). Validez y consistencia interna del inventario Maslach para Burnout en estudiantes de odontología de Cartagena, Colombia. Revista Colombiana de Psiquiatría. 46(2), 103-109.

2018, Vol. 11(22) 40-49 @The Author(s) 2018 Reprints and permission: www.americana.edu.co https://www.coruniamericana.edu.co/publicaciones/ojs/index.php/pensamientoamericano/index 